\title{
Treatment of peritoneal surface neoplasms with intraperitoneal chemotherapy in hyperthermia
}

\begin{abstract}
Effective treatment of peritoneal surface neoplasms is possible through the simultaneous use of cytoreductive surgery with intraperitoneal chemotherapy in hyperthermia. It is successfully performed in patients with peritoneal pseudomyxoma, mesothelioma, as well as a limited and resectable peritoneal carcinomatosis in the course of colorectal cancer. It can also be used in patients with gastric or ovarian cancer but also metastatic colorectal cancer or metastases to the ovaries from gastric cancer. Aggressive surgical management of patients with primary or secondary neoplasms of the peritoneal surface was initiated by Sugarbaker's research group.
\end{abstract}

Keywords: cytoreductive surgery, hyperthermic intraperitoneal chemotherapy, primary and secondary peritoneal surface malignancies.

DOI: $10.1515 /$ pjph-2015-0016

\section{INTRODUCTION}

The first cytoreductive surgery supplemented with intraperitoneal chemotherapy in hyperthermia was held at the Department of Surgical Oncology, Medical University of Lublin, on the 10th of November 2010 (Figure 1). It was performed in a patient with pseudomyxoma of peritoneum (lat. PseudoMyxoma peritonei; PMP) Since then, more than 200 patients were enrolled. The treatment with radical means was performed in nearly a half of them. Intraperitoneal treatment of peritoneal carcinomatosis (PC) in the course of cancers originating from gastrointestinal tract, female genital tract and primary peritoneal cancers (mesothelioma, PMP) is a promising method of therapy in oncology. PC occurs in nearly $15 \%$ of patients with colorectal cancer (CRC) and $40 \%$ of patients with gastric cancer (GC), and a substantial part of the population of patients with ovarian cancer (OC). The progression of the disease if not treated, always leads to intestinal obstruction, ascites, cachexia and eventually death of the patient. The efficacy of systemic chemotherapy in such cases is limited by the mucus-producing tumors. Metastases to the peritoneal surface are relatively resistant to cytotoxic drugs administered intravenously due to their scanty vascularization and the blood-peritoneum barrier. Combination of resection of macroscopically visible lesions throughout the peritoneal cavity (CytoReductive complete Surgery; CCRS) with simultaneous intraperitoneal chemotherapy in hyperthermia (Hyperthermic Intra-Peritoneal Chemotherapy; HIPEC), aimed at eliminating microscopic remnants of the tumor, would significantly improve treatment outcomes in patients with $\mathrm{PC}$.
HIPEC as a method of treatment for PC was first described in 1980, and in the following years popularized by Paul Sugerbaker [1,2].

The main advantages of HIPEC include:

1. to provide the appropriate dose to overcome drug resistance;

2. to ensure optimal conditions for uniform distribution of cytotoxic drugs in the peritoneal cavity;

3. treatment in hyperthermia.

The selective effect of hyperthermia on cancerous cells and its ability to enhance the effectiveness of chemotherapeutic agents is a valuable supplement to chemotherapy in the treatment of PC [3].

The effectiveness of HIPEC strictly depends on the proper qualification based on a quantitative assessment of the stage using the peritoneal carcionomatosis index (PCI). It is calculated and given as a score ranging from 0 to 39 points, which describes the size of all the lesions throughout the peritoneal cavity divided into 13 regions. Complete resection of the tumor lesions has a significant impact on long-term survival of patients with PC. The completeness of cytoreduction (CCR) is defined in the 4 points scale: 0 - complete removal of all the tumor tissues; 1 - remaining tumor foci do not exceed $2.5 \mathrm{~mm}$ (total cytoreduction, full penetration of cytotoxic drugs into tumor tissue); 2 - lesions in size from $2.5 \mathrm{~mm}$ to $2.5 \mathrm{~cm}$ - incomplete cytoreduction (moderate residual disease); 3 - remaining lesions larger than $2.5 \mathrm{~cm}$ (macroscopic residual disease). The scope of cytoreductive surgery depends on the location of the PC foci and consists of resection of peritoneum in each anatomical region (Table 1) [4]. 
TABLE 1. Peritonectomy procedures and resections, that are complete a cytoreduction procedure.

\begin{tabular}{cc}
\hline \hline PERITONECTOMY & RESCTIONS \\
\hline $\begin{array}{c}\text { Anterior parietal } \\
\text { peritonectomy }\end{array}$ & $\begin{array}{c}\text { Old abdominal incisions, } \\
\text { umbilicus end epigastric fat pad. }\end{array}$ \\
\hline Left upper quadrant peritonectomy & Greater omentectomy and splen \\
\hline $\begin{array}{c}\text { Right upper quadrant } \\
\text { peritonectomy }\end{array}$ & $\begin{array}{c}\text { Tumor on Glisson's capsule } \\
\text { of the liver }\end{array}$ \\
\hline Pelvic peritonectomy & Uterus, ovaries and rectosigmoid \\
\hline Omental bursectomy & Gallbladder and lesser omentum \\
\hline
\end{tabular}

\section{RESULTS}

Pseudomyxoma peritonei is a rare neoplasm (1-2 per million per year). It is characterized by the formation of large amounts of mucous and gelatinous contents in the abdominal cavity, spreading over an area of peritoneal surface, not through blood or lymphatic vessels. In more than half of the cases, PMP originates from the appendix but it can also occur in the course of mucosal cancers of the colon, ovaries, pancreas, bile ducts. The traditional procedure, involving repeated cytoreductive surgery with subsequent systemic chemotherapy is not satisfactory. 10-year survival rates ranges from 21 to $32 \%$, and the recurrence of the disease is observed in nearly $90 \%$ of patients $[5,6]$. The use of cCRS + HIPEC (with mitomycin C) allows to obtain many long-term survival rates in the majority of patients (10-year survival approx. 60\%-70\%) [7-9]. Sugarbaker et al. analyzed 385 patients diagnosed with PMP, treated with cCRS (CCR0/CCR1) and HIPEC. The overall 5-year survival rate was $86 \%$ [10]. In patients with peritoneal mesothelioma (mesothelioma peritonei) cCRS in combination with HIPEC 5-year survival rates range from $40 \%$ to $60 \%$ [11-13]. The results largely depend on the extent and completeness of the cytoreductive surgery preceding intraperitoneal chemotherapy.

PC is diagnosed in $10-15 \%$ patients with CRC. Relapses confined to the peritoneal surface concerns approximately $10-15 \%$ of patients. In over a half of patients the disease is limited to the peritoneal cavity, and 2/3 of these lesions are limited to one quadrant of the abdomen [14]. Verwal et al., conducted a randomized clinical trial in 105 patients, comparing cCRS + HIPEC with conventional treatment of PC. In the experimental group, $37 \%$ of patients underwent cCRS and nearly half of patients had a complete cytoreduction (residual disease less than $2.5 \mathrm{~mm}$ ).

HIPEC was performed with an open technique (Colosseum), using mitomycin $\mathrm{C}$ at $40^{\circ} \mathrm{C}$ for 90 minutes. After a median follow-up of 22 months, the median survival was 13 months in a conventional treatment group compare to 22 months in the experimental group. The results of this, so far only, randomized studies have recently been updated after 8 years of follow-up [15].

Five-year survival rates were $45 \%$, in patients treated with complete cytoreductive surgery and HIPEC. The results of this study clearly indicate that the cCRS supplemented with HIPEC significantly prolongs survival in patients with PC in the course of CRC.

An example of the possibilities of modern treatment of patients with macroscopic PC (without extra abdominal manifestation of the disease) in the course of CRC is complete cytoreduction and HIPEC with oxaliplatin. It should be noted that patients qualified to this method of treatment were in good general condition, up to the age of 65 years, with no evidence of bowel obstruction and clinically or radiologically apparent PC. There were no postoperative deaths reported and grade 2-3 toxicity was observed in $37 \%$ of patients. At a median follow-up of 55 months, in $73 \%$ of patients relapse was found after 14 months. $37 \%$ of patients suffered from peritoneal relapse (also combined with distant metastases). Patients with unresectable relapse received systemic chemotherapy. The rates of overall survival at 2,3 and 5 years were $73 \%, 53 \%$ and $49 \%$, respectively, and diseasefree survival rates were $48 \%, 42 \%$ and $34 \%$, respectively. The median survival was 60 months [16].

In their study, Chua et al., analyzed 2492 patients with PC in the course of CRC. 1084 patients were treated with cCRS + HIPEC. Overall survival ranged from 20 to 63 months, median 33 months and five-year survival rates ranged from $17 \%$ to $51 \%$, median $40 \%$. Overall survival of 1408 patients who underwent palliative surgery and / or chemotherapy ranged from 5 to 24 months and the median was 12.5 months. 5-year survival rates range from $13 \%$ to $22 \%$, median $13 \%$ [17]. The risk of complications in patients with esophageal cancer and pancreatic cancer is comparable to the complication rate in patients treated with the cCRS with HIPEC for PC the course of CRC, however long-term results are significantly improved [18]. After HIPEC + cCRS patients should be considered for further systemic therapy. It is particularly important that the qualification for the treatment with HIPEC was done at the earliest stage of PC, and not as a salvage therapy.

Advanced GC has a very poor prognosis. In $10-20 \%$ of patients potentially eligible for radical resection, and in $20-50 \%$ of patients after resection of GC, PC is diagnosed. The role of cCRS + HIPEC in the treatment of PC in the course of $\mathrm{GC}$ remains the subject of discussion. One of the first studies was published by Fujimoto et al., in 1988. The median survival in the group of treated patients was 7.2 \pm 4.6 months [19]. Similar results (median overall survival: 6.1-10.1 months) were published by other authors [19-21]. Yang et al., published the results of a randomized trial comparing cCRS + HIPEC and cCRS alone. The combination of cytoreductive surgery with HIPEC improved median overall survival by 4.5 months (11 vs 6.5 months) [22]. Indications regarding preventive HIPEC procedure

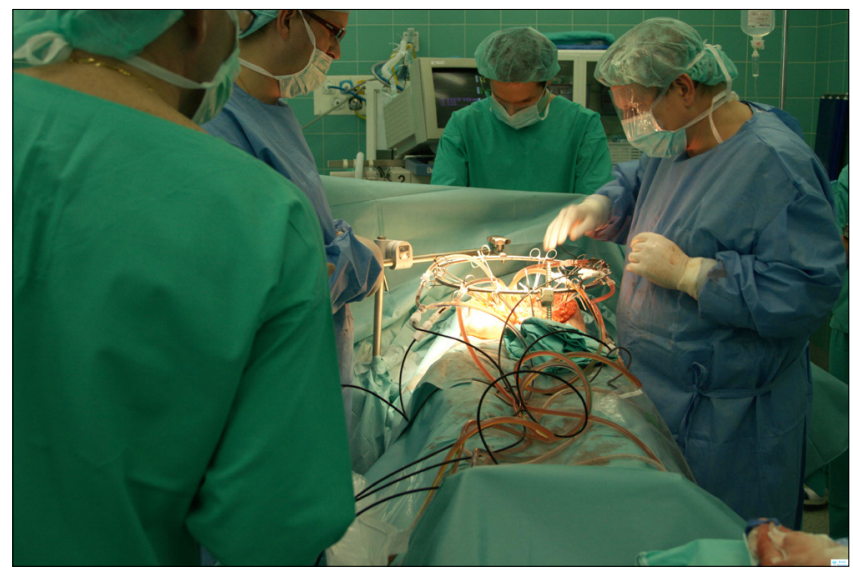

FIGURE 1. The first cytoreductive surgery supplemented with intraperitoneal chemotherapy in hyperthermia. 
in patients with high risk of $\mathrm{PC}$ in the course of $\mathrm{GC}$ not clear. However this technique can be considered in patients with positive peritoneal washing cytology, as studies show an improvement in overall survival in this clinical situation [23].

Despite the improvements in the treatment of $\mathrm{OC}$ in the last decade, treatment results in an advanced stage of the disease unsatisfactory. Surgical treatment involving resection of all visible tumor lesions in the abdominal cavity with complementary chemotherapy is the main method of treatment. Effectiveness of cCRS + HIPEC in patients with relapse after first or second line of systemic treatment was confirmed in numerous cohort studies and phase II trials. The rates of 5 -year survival ranges from $16 \%$ to more than $50 \%$, which is significantly improved in comparison to patients receiving only chemotherapy [24-27].

\section{Contraindications for the use of HIPEC}

Absolute contraindications for HIPEC include: poor overall condition (Karnofsky performance status $<70$ ), unresectable extra abdominal metastases, the inability to obtain cCRS (invasion of mesenteric vessels, celiac trunk, the aorta, diffuse infiltration of retroperitoneal space), which usually indicates that the $\mathrm{PC}$ is advanced and disseminated. Relative contraindications are progression of $\mathrm{PC}$ and/or the inability to eliminate ascites during preoperative systemic chemotherapy, which usually indicates the presence of unresectable lesions, multiple metastases to the liver, progression after neoadjuvant chemotherapy [28]. In case of limited, resectable metastases from colorectal cancer to the liver, the prognosis is not significantly inferior as compared to patients without metastases [29].

\section{CONCLUSION}

In recent decades, significant progress in the treatment of patients with primary and secondary neoplasms of the peritoneal surface has been observed. So far, patients with diagnosis of PC have been reffered to supportive treatment or palliative chemotherapy. Early detection of PC followed by radical surgical resection in conjunction with HIPEC, allows for achieving optimum treatment results. However, there is a need for further research on the use of HIPEC as PC prophylaxis in patients with high risk of this route of cancer spreading. Only palliative treatment of limited PC in the course of colorectal, ovarian and appendix is insufficient.

Treatment of PC must be multidisciplinary, involving oncological surgeon (complete cytoreductive surgery; cCRS), clinical oncologist (selection of cytostatic doses used intraperitoneally), perfusionist (supervision over the operation of the peritoneal perfusion system and controlled hyperthermia) and anesthesiologist (safe anesthesia during multiorgan resections).

\section{REFERENCES}

1. Spratt JS, Adcock RA, Muskovin M, et al. Clinical delivery system for intraperitoneal hyperthermic chemotherapy. Cancer Res. 1980;40(2):256-60.

2. Sugarbaker PH, Graves T, DeBruijn EA, et al. Early postoperative intraperitoneal chemotherapy as an adjuvant therapy to surgery for peritoneal carcinomatosis from gastrointestinal cancer: pharmacological studies. Cancer Res. 1990;50(18):5790-4.

3. de Bree E, Romanos J, Tsiftsis DD. Hyperthermia in anticancer treatment. Eur J Surg Oncol. 2002;28(1):95.

4. Sugarbaker PH. Peritonectomy procedures. Ann Surg. 1995;221(1):2942.

5. Miner TJ, Shia J, Jaques DP, et al. Long-term survival following treatment of pseudomyxoma peritonei: an analysis of surgical therapy. Ann Surg. 2005;241(2):300-8.

6. Ronnett BM, Zahn CM, Kurman RJ, et al. Disseminated peritoneal adenomucinosis and peritoneal mucinous carcinomatosis. A clinicopathologic analysis of 109 cases with emphasis on distinguishing pathologic features, site of origin, prognosis, and relationship to "pseudomyxoma peritonei”. Am J Surg Pathol. 1995;19(12):1390-408.

7. Chua TC, Moran BJ, Sugarbaker PH, et al. Early- and long-term outcome data of patients with pseudomyxoma peritonei from appendiceal origin treated by a strategy of cytoreductive surgery and hyperthermic intraperitoneal chemotherapy. J Clin Oncol. 2012;30(20):2449-56.

8. Cole KL, Choudry HA, Jones HL, et al. Critical role of hyperthermic intraperitoneal chemoperfusion in the treatment of a patient with Pseudomyxoma peritonei. J Surg Oncol. 2012;106(4):513-6.

9. Sorensen O, Flatmark K, Reed W et al. Evaluation of complete cytoreductive surgery and two intraperitoneal chemotherapy techniques in pseudomyxoma peritonei. Eur J Surg Oncol. 2012;38(10):969-76.

10. Sugarbaker PH, Chang D. Results of treatment of 385 patients with peritoneal surface spread of appendiceal malignancy. Ann Surg Oncol. 1999;6(8):727-31.

11. Yan TD, Deraco M, Baratti D et al. Cytoreductive surgery and hyperthermic intraperitoneal chemotherapy for malignant peritoneal mesothelioma: multi-institutional experience. J Clin Oncol. 2009;27(36):623742.

12. Baratti D, Kusamura S, Cabras AD, Deraco M. Cytoreductive surgery with selective versus complete parietal peritonectomy followed by hyperthermic intraperitoneal chemotherapy in patients with diffuse malignant peritoneal mesothelioma: a controlled study. Ann Surg Oncol. 2012;19(5):1416-24.

13. Yan TD, Black D, Sugarbaker PH et al. A systematic review and meta-analysis of the randomized controlled trials on adjuvant intraperitoneal chemotherapy for resectable gastric cancer. Ann Surg Oncol. 2007;14(10):2702-13.

14. Chu DZ, Lang NP, Thompson C et al. Peritoneal carcinomatosis in nongynecologic malignancy. A prospective study of prognostic factors. Cancer. 1989;63(2):364-7.

15. Verwaal VJ, Bruin S, Boot H, et al. 8-year follow-up of randomized trial: cytoreduction and hyperthermic intraperitoneal chemotherapy versus systemic chemotherapy in patients with peritoneal carcinomatosis of colorectal cancer. Ann Surg Oncol. 2008;15(9):2426-32.

16. Elias D, Gilly F, Boutitie F et al. Peritoneal colorectal carcinomatosis treated with surgery and perioperative intraperitoneal chemotherapy: retrospective analysis of 523 patients from a multicentric French study. J Clin Oncol. 2010;28(1):63-8.

17. Chua TC, Esquivel J, Pelz JO, Morris DL. Summary of current therapeutic options for peritoneal metastases from colorectal cancer. J Surg Oncol. 2013;107(6):566-73.

18. Chua TC, Yan TD, Saxena A, Morris DL. Should the treatment of peritoneal carcinomatosis by cytoreductive surgery and hyperthermic intraperitoneal chemotherapy still be regarded as a highly morbid procedure?: a systematic review of morbidity and mortality. Ann Surg. 2009;249(6):900-7.

19. Fujimoto S, Shrestha RD, Kokubun M et al. Intraperitoneal hyperthermic perfusion combined with surgery effective for gastric cancer patients with peritoneal seeding. Ann Surg. 1988;208(1):36-41.

20. Loggie BW, Fleming RA, McQuellon RP, et al. Cytoreductive surgery with intraperitoneal hyperthermic chemotherapy for disseminated peritoneal cancer of gastrointestinal origin. Am Surg. 2000;66(6):561-8.

21. Glehen O, Gilly FN, Arvieux C, et al. Peritoneal carcinomatosis from gastric cancer: a multi-institutional study of 159 patients treated by cytoreductive surgery combined with perioperative intraperitoneal chemotherapy. Ann Surg Oncol. 2010;17(9):2370-7. 
22. Yang XJ, Huang CQ, Suo T, et al. Cytoreductive surgery and hyperthermic intraperitoneal chemotherapy improves survival of patients with peritoneal carcinomatosis from gastric cancer: final results of a phase III randomized clinical trial. Ann Surg Oncol. 2011;18(6):1575-81.

23. Roviello F, Caruso S, Neri A, Marrelli D. Treatment and prevention of peritoneal carcinomatosis from gastric cancer by cytoreductive surgery and hyperthermic intraperitoneal chemotherapy: overview and rationale. Eur J Surg Oncol. 2013;39(12):1309-16.

24. Spiliotis J, Vaxevanidou A, Sergouniotis F, et al. The role of cytoreductive surgery and hyperthermic intraperitoneal chemotherapy in the management of recurrent advanced ovarian cancer: a prospective study. J BUON. 2011;16(1):74-9.

25. Munoz-Casares FC, Rufian S, Rubio MJ, et al. The role of hyperthermic intraoperative intraperitoneal chemotherapy (HIPEC) in the treatment of peritoneal carcinomatosis in recurrent ovarian cancer. Clin Transl Oncol. 2009;11(11):753-9.

26. Bae JH, Lee JM, Ryu KS et al. Treatment of ovarian cancer with paclitaxel- or carboplatin-based intraperitoneal hyperthermic chemotherapy during secondary surgery. Gynecol Oncol. 2007;106(1):193-200.

27. Ryu KS, Kim JH, Ko HS, et al. Effects of intraperitoneal hyperthermic chemotherapy in ovarian cancer. Gynecol Oncol. 2004;94(2):325-32.

28. Elias D, Benizri E, Vernerey D et al. Preoperative criteria of incomplete resectability of peritoneal carcinomatosis from non-appendiceal colorectal carcinoma. Gastroenterol Clin Biol. 2005;29(10):1010-3.

29. Varban O, Levine EA, Stewart JH et al. Outcomes associated with cytoreductive surgery and intraperitoneal hyperthermic chemotherapy in colorectal cancer patients with peritoneal surface disease and hepatic metastases. Cancer. 2009;115(15):3427-36.

\section{Corresponding author}

Jerzy Mielko

11 Staszica Str., 20-081 Lublin

E-mail: jerzy.mielko@umlub.pl

Photographic documentation supplied by the Authors. 